\title{
La traduction française des Recherches sur la méthode de Carl Menger (1883-2011)
}

Die französische Übersetzung von Untersuchungen über die Methode von Carl Menger (1883-2011)

The French translation of Investigations upon the Methods by Carl Menger (1883-2011)

\section{Jean Magnan de Bornier}

\section{OpenEdition}

\section{Journals}

Édition électronique

URL : https://journals.openedition.org/austriaca/1475

DOI : 10.4000 /austriaca. 1475

ISSN : 2729-0603

\section{Éditeur}

Presses universitaires de Rouen et du Havre

\section{Édition imprimée}

Date de publication : 1 juin 2020

Pagination : 215-219

ISBN : 979-10-240-1492-0

ISSN : 0396-4590

Référence électronique

Jean Magnan de Bornier, «La traduction française des Recherches sur la méthode de Carl Menger (1883-2011) », Austriaca [En ligne], 90 | 2020, mis en ligne le 01 juin 2021, consulté le 06 octobre 2022. URL : http://journals.openedition.org/austriaca/1475; DOI : https://doi.org/10.4000/austriaca.1475

Ce document a été généré automatiquement le 6 octobre 2022.

Tous droits réservés 


\title{
La traduction française des Recherches sur la méthode de Carl Menger (1883-2011)
}

\author{
Die französische Übersetzung von Untersuchungen über die Methode von \\ Carl Menger (1883-2011) \\ The French translation of Investigations upon the Methods by Carl Menger \\ (1883-2011)
}

Jean Magnan de Bornier

1 La traduction des Recherches sur la méthode par Gilles Campagnolo permettra à beaucoup de prendre enfin connaissance, dans notre langue, de ce texte fondamental de Carl Menger ${ }^{1}$, le second de ses ouvrages majeurs après les Principes d'économie politique, dont une traduction par le même traducteur a paru au début de l'année $2020^{2}$.

2 Auteur de nombreux articles et ouvrages consacrés au fondateur de l'école autrichienne, Campagnolo offre dans ce volume non seulement une version limpide et une lecture agréable des Untersuchungen, mais aussi deux essais approfondis autour des textes de Menger, destinés à guider le lecteur dans le livre ainsi que dans le méandre des controverses qui l'ont entouré. Le premier de ces essais reprend et commente le contenu de ces Recherches, alors que le second retrace les débats qu'il a suscités dans le monde germanique, connus comme constituant la « querelle des méthodes».

3 C'est bien évidemment des Principes de Menger que tout découle historiquement. Publiés en 1871, ces Grundsätze der Volkswirtschaftslehre introduisent une nouvelle approche de la compréhension du monde économique et constituent un des éléments fondateurs de la révolution marginaliste, avec les contributions contemporaines de Stanley Jevons et Léon Walras. Mais cette première contribution de Menger ne satisfait pas le monde universitaire germanique, qui lui reproche en particulier sa méthode abstraite et anhistorique. C'est face à cette réaction mi-indifférente mi-hostile que Menger prépare et publie les Untersuchungen, texte dans lequel non seulement il justifie (de manière magistrale!) sa méthode, mais où il attaque également de front l'école 
historique, dominante en Allemagne. C'est alors le début de la querelle des méthodes ou Methodenstreit, au cours de laquelle les chefs de file des courants opposés, Gustav Schmoller et Menger, vont s'affronter sans merci.

La lecture des Untersuchungen ne montre pourtant en aucun cas un Menger sectaire ou ennemi de l'histoire ; elle révèle plutôt un auteur qui entend remettre chaque partie et chaque méthode particulière à sa juste place. C'est toute l'importance de la classification des sciences sociales en trois groupes distincts, à savoir les sciences historiques, les sciences théoriques et les sciences pratiques. L'intérêt des sciences théoriques dans cet ensemble, selon Menger, consiste en ce qu'elles (et elles seules) fournissent les relations causales nécessaires à la compréhension des phénomènes se déroulant sous les yeux de tout un chacun, et pour lesquels l'économiste cherche à proposer des interprétations. C'est par la recherche des Realtypen, des relations élémentaires entre éléments de l'analyse, que le théoricien pourra fournir les briques de cette compréhension. Et ce type réel (qui préfigure l'idéal-type de Max Weber) met en relation les unités élémentaires, les agents économiques; il ne peut pas être explicité en termes d'agrégats : l'individualisme méthodologique, qui n'a pas encore reçu son nom, est là exposé pour la première fois de manière systématique.

5 Une autre classification proposée par Menger est celle des «orientations» de la recherche dans les sciences sociales. En croisant le point de vue du chercheur (individuel ou général) et la nature du phénomène étudié (individuel ou collectif), on obtient quatre orientations possibles (voir le tableau $1 \mathrm{du}$ premier texte de Campagnolo ${ }^{3}$ ), dont l'une est hors de portée de l'activité scientifique selon Menger: l'orientation générale et collective qu'on peut traduire par étude théorique des agrégats pour en donner une idée rapide. Et pourtant, tel était le projet scientifique de l'école historiciste! En revanche, Menger ne voit aucune objection aux monographies qui portent sur de vastes ensembles, à condition évidemment qu'on ne prétende pas à partir d'elles tirer des lois générales.

6 Un autre point focal du livre de Menger est son approche duale des institutions sociales ou "produits sociaux ». Il distingue en effet celles qui sont le résultat délibéré des actions humaines - à travers des conventions ou une législation - de celles qui sont «le résultat non réfléchi du développement sociétal». Les premières sont «d'origine pragmatique », alors que les secondes sont «d'origine organique ». L'institution de la monnaie, sur laquelle Menger fournit des développements dès les Principes (chapitre 8), et qu'il développe amplement dans ses réflexions par la suite, est un exemple notable pour lui de développement organique.

7 En lisant le chapitre consacré aux produits sociaux, les ordres construits par contraste avec les ordres spontanés de Friedrich A. Hayek viennent immédiatement à l'esprit ; mais Menger utilise cette dualité pragmatique-organique à titre classificatoire, alors que Hayek, on le sait, insiste sur la supériorité des ordres spontanés. À lire Menger cependant, on peut difficilement douter de sa grande influence sur la pensée de Hayek dans ce domaine, même si ce dernier a préféré bien souvent magnifier celle des philosophes des Lumières écossaises à ce propos.

8 Le texte de Menger, clairement, peut être lu en lui-même, sans l'aide d'un guide. Pourtant Gilles Campagnolo (auteur de cette précieuse traduction) et la maison d'édition ont jugé bon de l'accompagner de deux essais qui encadrent matériellement celui de Menger. Ces essais présentent une richesse indéniable à trois points de vue cumulatifs. 
Ils fournissent d'abord d'amples informations contextuelles : qui était Menger, d'où il venait, familialement et professionnellement, quel était l'état de l'économie universitaire en Autriche et en Allemagne dans la seconde moitié du XIXe siècle ; puis, s'agissant de la "querelle des méthodes ", une relation précise des événements, voire des micro-événements, qui constituent le substratum dans lequel les écrits apparaissent. Ces informations contextuelles, dûment commentées, permettent au lecteur une perception bien plus aiguë des textes, y compris s'il en prend connaissance, comme nous l'avons fait, après lecture desdits textes.

10 Deuxièmement, Campagnolo fournit des résumés ou plutôt des réexpositions d'une remarquable clarté du texte mengerien. De même qu'on peut évidemment lire ce volume en se contentant du texte de Menger, on pourrait presque se contenter du premier essai (Présentation) pour en saisir l'essentiel à moindre investissement (tel n'est cependant pas notre conseil!)

11 Troisièmement, les essais de Campagnolo ajoutent de la substance aux arguments de Menger, et cela pour deux raisons simples : en premier lieu, il tient compte de tous les débats qui, depuis environ cent trente ans, ont porté sur l'interprétation à donner des thèses mengeriennes, et plus généralement autrichiennes; et, en second lieu, Campagnolo commente Menger autant en philosophe qu'en économiste, allant d'ailleurs jusqu'à faire de Menger l'inventeur d'une forme de philosophie économique, peut-être même de la philosophie économique.

12 La question posée dans la section 5 de la Présentation, celle du "projet théorique » de Menger, est bien de celles qui permettent un élargissement de la vision du lecteur. En naviguant à la suite de Tony Lawson entre les positions concernant trois concepts fondamentaux, à savoir déductivisme, réalisme, causalité, Campagnolo situe Menger comme adhérant à ces trois options. Cette position contraste ainsi avec celle d'un hypothétique Menger non réaliste, que croient voir certains de ses critiques comme Karl Milford, ou avec les positions du positivisme friedmanien (ni réaliste ni causaliste).

13 Une autre direction de l'interprétation de Campagnolo est son insistance sur ce qu'on peut nommer la «neutralité ontologique» de Menger. On a l'habitude de bien distinguer l'individualisme méthodologique de Menger de l'individualisme politique, plaidoyer pour une société valorisant plus ou moins exclusivement les actions et les réalisations individuelles, sans se préoccuper de la dimension collective des actions ni des valeurs qui leur sont liées. Mais Campagnolo préfère le contraster avec un individualisme ontologique, position philosophique qui affirmerait la seule réalité des individus. C'est l'écueil d'un amalgame entre individualisme méthodologique et individualisme ontologique que Menger aurait su éviter dans sa lutte contre l'utilisation des Kollektivbegriffe (concepts collectifs). Cette interprétation originale de l'individualisme mengerien, inhabituelle et un rien provocatrice, nous semble mériter de plus amples débats. Elle n'empêche d'ailleurs pas Campagnolo d'insister sur la stricte neutralité de Menger en matière de choix de système économique, ce qui lui confère une place originale à l'intérieur de l'école qu'il a initiée, l'école autrichienne.

14 On ne fera pas la liste de tous les développements intéressants des essais de Campagnolo, mais ceux concernant la classification des sciences sociales et la mathématisation de l'économie nous ont paru très pertinents.

15 Relire le Menger des Untersuchungen à la lueur des discussions de méthodologie et de philosophie d'aujourd'hui est particulièrement roboratif. Y mêler la lecture des essais 
de Campagnolo avant, pendant, ou après peut ajouter à cette expérience d'utiles compléments.

\section{NOTES}

1. Carl Menger, Recherches sur la méthode dans les sciences sociales et en économie politique en particulier, Paris, EHESS, "Translations », 2011: traduction française annotée présentée et commentée par Gilles Campagnolo des Untersuchungen über die Methode der Socialwissenschaften und der Politischen Ökonomie insbesondere, Leipzig, Duncker \& Humblot, 1883, $286 \mathrm{p}$.

2. Carl Menger, Principes d'économie politique, Paris, Seuil, 2020 : traduction française annotée, présentée et commentée par Gilles Campagnolo des Grundsätze der Volkswirtschaftslehre, Wien, 1871, Wilhelm Braumüller, 288 p. Voir l'information additionnelle apportée dans l'appendice du présent texte.

3. Carl Menger, Recherches sur la méthode dans les sciences sociales, op. cit., p. 49.

\section{RÉSUMÉS}

Auteur de nombreux articles et ouvrages consacrés au fondateur de l'école autrichienne, Gilles Campagnolo offre en français une version limpide et d'une lecture agréable non seulement des Recherches sur la méthode de Carl Menger (Untersuchungen über die Methode der Socialwissenschaften und der politischen ökonomie insbesondere), mais aussi deux essais approfondis autour des textes de Menger, destinés à guider le lecteur dans le livre ainsi que dans le méandre des controverses qui l'ont entouré. Le premier de ces essais reprend et commente le contenu des Recherches de Menger, alors que le second retrace les débats qu'il a suscités dans le monde germanique, connus comme constituant la "querelle des méthodes»(Methodenstreit). La traduction de Gilles Campagnolo permettra à beaucoup de prendre enfin connaissance, dans notre langue, de ce texte fondamental de Carl Menger, le second de ses ouvrages majeurs après les Principes d'économie politique, dont une traduction par le même traducteur vient de paraître au Seuil (2020).

Als Autor von zahlreichen Artikeln und Werken über den Gründer der Österreichischen Schule, legt Gilles Campagnolo unter dem Titel Recherches sur la méthode in angenehm lesbarer Form eine klare französische Übersetzung von Carl Mengers Untersuchungen über die Methode der Socialwissenschaften und der politischen Ökonomie insbesondere (Leipzig, Dunckler \& Humblot, 1883, 2885.) vor und darüberhinaus zwei fundierte Essays im Zusammenhang mit den Texten Mengers, welche dem Leser eine Hilfestellung für das Lesen des Werkes geben und über die Kontroversen informieren, die seine Veröffentlichung ausgelöst hat. Der erste Essay nimmt den Inhalt der Recherches wieder auf und kommentiert ihn ausführlich, während der zweite die Debatten nachzeichnet, die das Buch im deutschsprachigen Raum auslösten und die als konstitutiver Teil des „Methodenstreites“ bekannt sind. Die Übersetzung von Gilles Campagnolo 
wird es zahlreichen Personen ermöglichen, diesen grundlegenden Text von Carl Menger endlich in französischer Sprache kennen zu lernen; es ist das zweite seiner Hauptwerke ; das erste sind die Grundsätze der Volkswirtschaftslehre (Wien, 1871), die erste französische Übersetzung dieses Werkes, ebenfalls von G.Campagnolo, ist vor kurzem bei den Editions du Seuil erschienen (Principes d'économie politique, Paris, 2020).

Gilles Campagnolo has authored many essays and books on the founder of the Austrian School, Carl Menger. With the first French translation of Menger's 1883 Investigations upon the Methods of Social Sciences and Political Economy in particular (Untersuchungen über die Methode der Socialwissenschaften und der politischen Ökonomie insbesondere 1883, Leipzig, Dunckler \& Humblot), he provides the French readership with a faithful and crystal-clear volume, long awaited and pleasant to read. Two highly recommendable essays by Campagnolo flank his 300 p. translation: an information-rich presentation of Menger in Vienna and a detailed account of the intricate debate known as the "Dispute over the Methods" (Methodenstreit) in the German-speaking world around 1880s-1900s (Menger's Investigations were his stake in the latter). Campagnolo's translations fill a blatant lacuna in the French language scholarship as far as the works by Menger are concerned. The first French version (by the same) of the grounding work Principles of Political Economy (Grundsätze der Volkswirtschaftslehre, 1871, Vienna) has just been published (Principes d'économie politique, Paris, Seuil, 2020).

\section{INDEX}

Mots-clés : Menger (Carl), méthode

Keywords : Menger (Carl), method

Schlüsselwörter : Menger (Carl), methode

\section{AUTEUR}

\section{JEAN MAGNAN DE BORNIER}

Aix-Marseille Université 